\title{
Behaviour of a puff of resuspended sediment: a conceptual model
}

\author{
J.C. Brun-Cottan ${ }^{\mathrm{a}, *}$, S. Guillou ${ }^{\mathrm{b}}$, Z.H. Li ${ }^{\mathrm{c}}$ \\ ${ }^{a}$ UPRESA-6143 Morphodynamique Continentale et Côtière CNRS/Universités de Caen et de Rouen, 24 rue des Tilleuls, 14000 Caen, France \\ ${ }^{\mathrm{b}}$ LUSAC, Site Universitaire de Cherbourg, BP-78, 50130, Octeville, France \\ ${ }^{\mathrm{c}}$ Institute of Mechanics, Chinese Academy of Sciences, 15 Zhong Guan Cun Road, Beijing, People's Republic of China
}

Received 11 February 1999; accepted 3 March 2000

\begin{abstract}
The particulate matter concentration above the seabed is usually assumed to decrease with height, following an exponential or Rouse profile. Many particulate matter concentration profiles with a peak were found on the North Mediterranean bottom water at a few tens of metres above the bottom. A particle size signal at the same altitude was found in this area and on the New York Bight shelf. It is assumed that this unexpected shape is due to a cloud of resuspended cohesive sediments originating from an impulse resuspension process. A simplified three-dimensional numerical model is proposed to describe the behaviour of resuspended particulate matter that originates from a sediment impulse vertically injected in the bottom water. This model reproduces the concentration profile shape observed, and it gives indications concerning the length and time characteristics of such a cloud, depending on the water velocity and bottom boundary layer properties. () 2000 Elsevier Science B.V. All rights reserved.
\end{abstract}

Keywords: Sediment; Sediment resuspension; Cohesive sediment; Nepheloid layer

\section{Introduction}

Usually, the concentration of particulate matter (PM) above the sea bottom is expected to decrease with distance from the bottom, following an exponential law (Biscaye and Eittreim, 1977) or Rouse profile. This is because the steady state hypothesis implies the balance, at every height above the bottom, of the gravitational settling flux and the vertical mixing flux. Some vertical concentration profiles showing a non-exponential shape with a maximum concentration value at several metres above the bottom were found in North Western Mediterranean. This shape was found during the PHYCEMED 1987 programme

\footnotetext{
* Corresponding author.

E-mail address: brun-cottan@geos.unicaen.fr (J.C. BrunCottan).
}

(Fig. 1) and the CYBELE cruise (April/May 1990) (Fig. 2).

Particle size distribution (PSD) may also be a good marker of the resuspension processes; indeed, the sediment particle mean size is always larger than the PM one above the seabed, at steady state (BrunCottan, 1984). Some vertical particle mean size profiles present a maximum value, or bump, in the first tens of metres above the bottom in the New York Bight. This was observed at several locations during the RACACA 1980 cruise.

The hypothesis that the observed PM concentration or PM mean size bumps originate from the advective longitudinal transport of layers brought out from coastal waters, shelf waters or from isolated bathymetric accidents has a very poor probability of applying here. This is because the altitude above the bottom of these observed PM signals is always less 

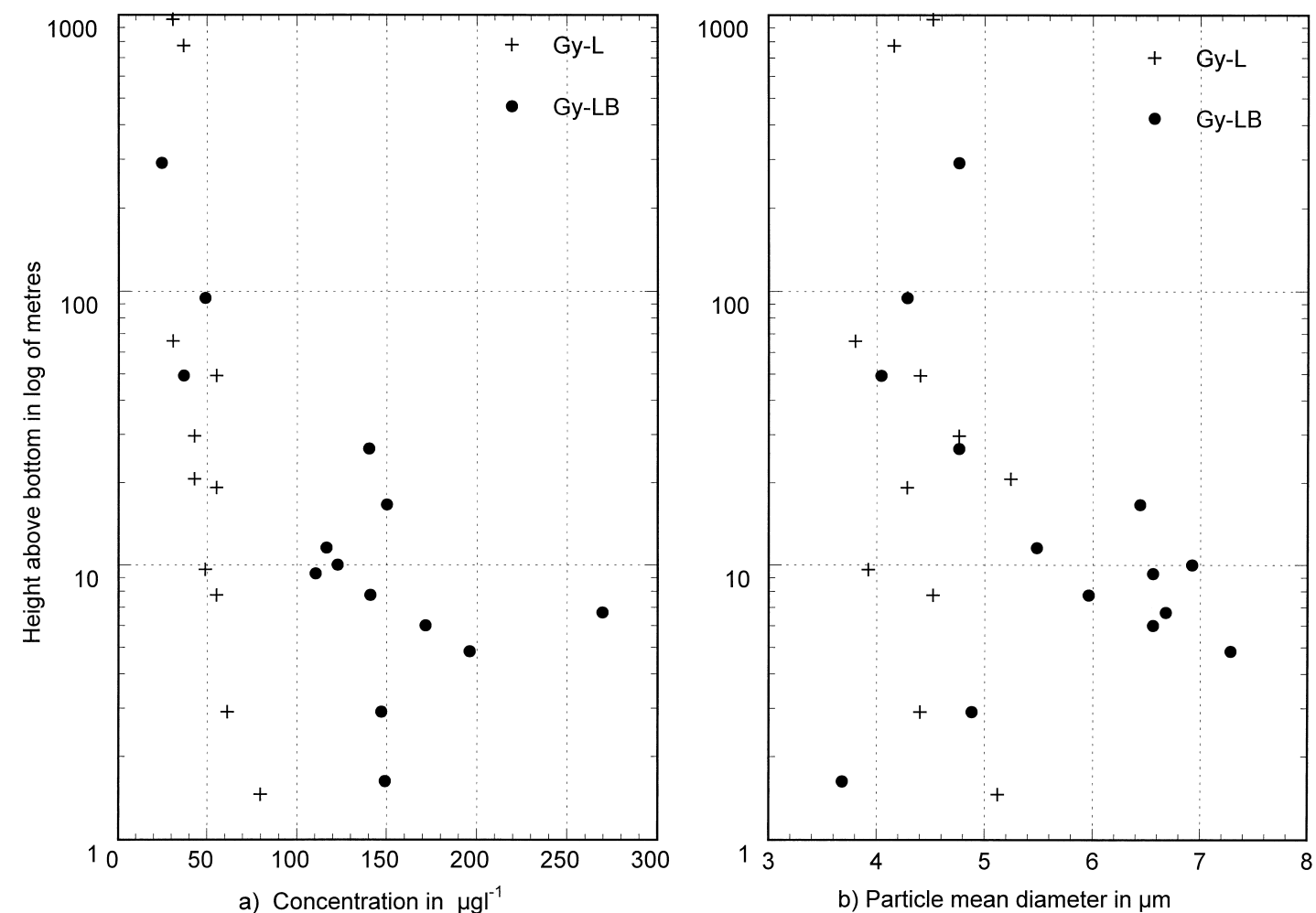

Fig. 1. Suspended particles in Northwestern Mediterranean (PHYCEMED cruise): mass concentration and mean size versus height above the seabed. The lag time between Gy-L and Gy-LB is 1 month. The water depth is $1950 \mathrm{~m}$.

than $50 \mathrm{~m}$ and the reported measurements were obtained above the flat bottom areas, at the considered phenomenon scale. They actually do not depend on seabed depth and roughness or distances from known PM sources. In a given location, they can be either present or not, depending on the moment of the observation. Moreover, the advective longitudinal transport (including the influence of some obstacles of few metres size) does not fit the time variability of the PM signal above the seabed. Signal variability reported in the New York Bight (Churchill et al., 1994) and in the South East area of Nova Scotia during the HEBBLE project at more than $4000 \mathrm{~m}$ depth (Hollister et al., 1984).

From the previous results, it is assumed here that the atypical concentration or particle's mean size profiles found during these cruises result from the behaviour of temporary clouds of resuspended cohesive sediment in the bottom water shear flow. Thus, the aim of this paper is to show that, in the mixed layer above the seabed, where the hydrodynamical condi- tions are at quasi-steady-state, a brief injection of bottom sediment can locally appear. This resuspension process is thought to be triggered by a local input of an excess energy in the system, at the scales of a few seconds and some tens of metres. These mechanisms may reflect local random fluctuations of the bottom velocity that exceed the critical erosion shear stress value. These increases of the bottom velocity can be provided by the propagation of energy from a variable intense wind field (down to the deep) or by any periodic or variable process such as tidal currents, internal waves, bottom fishing gear (Churchill et al., 1994). The considered initial puff of resuspended sediment moves and spreads, submitted to the water velocity field and the turbulent mixing mechanisms.

The possibility that spatially confined sediment resuspension can both produce Rouse (exponential) and bump profiles of PM concentration or mean size is tested here. This can be done by the numerical simulation of the transport and mixing of a spatially 


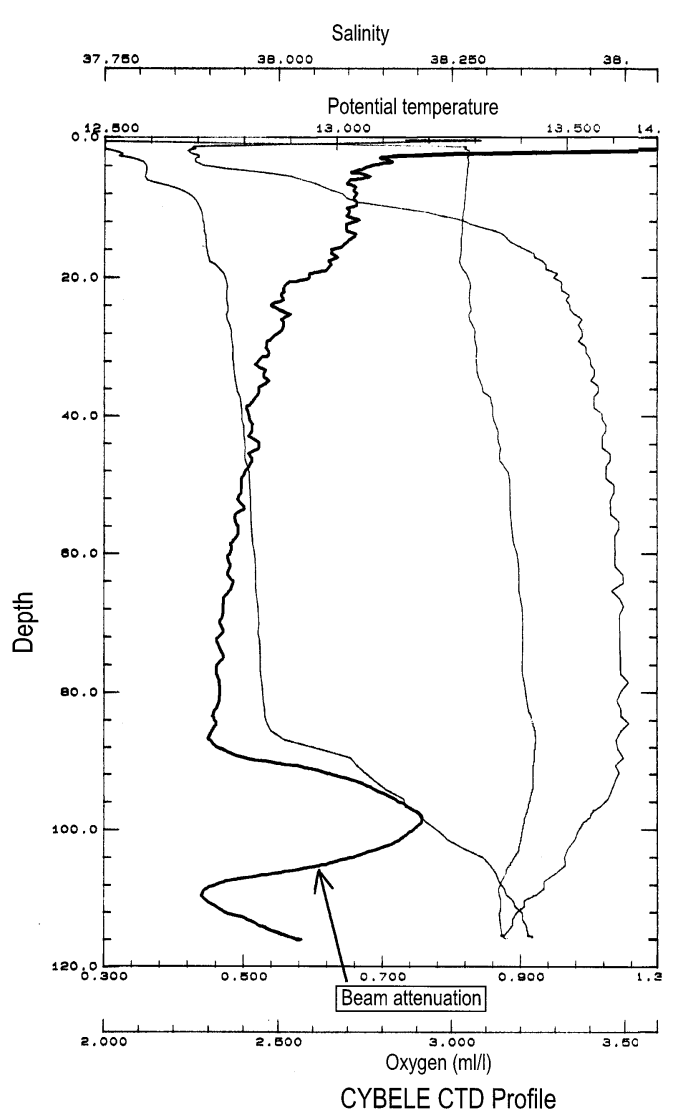

Fig. 2. CTD profile in the Gulf of Lion (CYBELE cruise, 1990, R/V Marion Dufresne). The beam attenuation was measured with a light transmissometer.

confined sediment cloud within the bottom boundary layer (BBL). The BBL is characterized by a sheared flow and high values of the horizontal and vertical mixing coefficients (Armi and Millard, 1976).

\section{Experimental data}

The PHYCEMED programme is located in the Gulf of Lions (Northwestern Mediterranean), above a flat bottom at $2000 \mathrm{~m}$ depth at a point midway between the French coast and Corsica. During this programme, many water samples were taken close to the seabed with a CTD/Rosette system (5-1 Niskin bottles) and the PM concentration was measured by weighting filtered samples. The PSD was determined with a Coulter Counter Multisizer equipped with two probes
(100 and $280 \mu \mathrm{m}$, giving a size spectrum memorized in $2 \times 256$ channels), which allows measuring the particle size ranging from 1.2 to $130 \mu \mathrm{m}$. The PSD parameters (mean, median, mode sizes and quadratic dispersion) were estimated by fitting a log-normal law (Brun-Cottan, 1986). Previous measurements in a given bottle have shown that repetitive Coulter measurements provide a dispersion of the PSD central parameters less than 5\%. The Gy-L station is far away from the shelf breaks of France and Corsica (more than $100 \mathrm{~km}$ ) and the seabed is very flat with a depth of $2000 \mathrm{~m}$. This station was occupied twice with an interval of one month (Fig. 1). The first time, the PM concentration and the mean particle size profiles corresponded to the classic exponential Rouse scheme, the wind was calm during the cast and the week before. On the second occasion, an increase as a bump of both the PM concentration and the particle mean size was observed at $10 \mathrm{~m}$ above the bottom.

The CYBELE cruise was done on the continental shelf break of the Gulf of Lions. During this cruise, both classical and bump PM concentration profiles were observed many times with a turbidimeter tied to a CTD/Rosette system (Fig. 2).

The RACACA cruise (R/V Conrad, LDEO of Columbia University) was located in the SEEP I experiment area in the waters of the Mid-Atlantic Bight shelf and slope south of Cape Code and Long Island. During the RACACA cruise, casts done in the low radon zone (i.e. high vertical mixing area) show small PM concentration near the bottom, but some PSD profiles, also obtained with a Coulter Counter system, show a maximum mean size around an altitude of 20/30 $\mathrm{m}$ above the bottom (Fig. 3). The PM mass concentration does not show maximum values at this altitude, probably because the particle size measurement system (Coulter Counter) is very sensitive and the intrusion of large and wet sediment particles can then have a significant impact on the PSD but not on the PM concentration (Perigaud et al., 1982). The proportion of bump profiles regarding the Rouse ones is nearly $10 \%$.

\section{The model and its mathematical expression}

The proposed idea of a local impulse-like resuspension process is tested here by the numerical simulation 


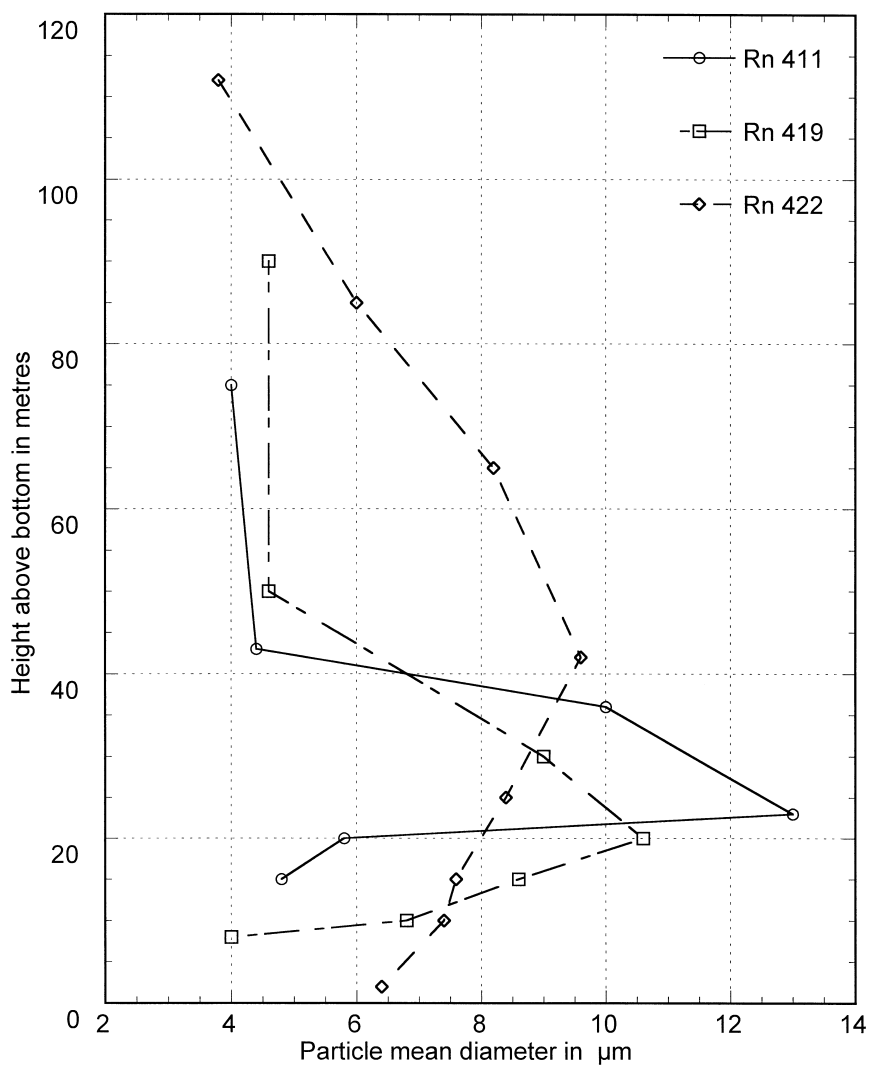

Fig. 3. Particle mean size diameters above the seabed in the New York Bight (shelf break). The three considered Rn stations were at depths ranging from 300 to $900 \mathrm{~m}$.

of the behaviour of a short vertical pulse of cohesive sediment in the BBL. The BBL is considered well developed with a rough bottom and with no viscous sub-layer. Thus, the water velocity and the turbulent mixing coefficients are calculated with the classical hydrodynamic methods.

Dispersion effect of a sheared velocity profile close to a wall was shown by Taylor (1921) and an analytical solution of the transport of particulate matter from steady state sources was proposed by Csanady (1973), particularly for elevated sources such as chimneys. Perigaud et al. (1982) have initiated the simulation of the distribution of brief sediment resuspension from a punctual source.

A single jet of cohesive sediment is assessed to be injected from a small bottom area for a short time in the water above the seabed. Thus, the spatial distribution of the resulting cloud of PM is simulated as a function of time, the development of the profile is studied and also the fraction of the cloud mass lost by the redeposition of the particles.

The gravity centre $G$ of the initial pulse of particulate matter is located at the distances $x=x_{0}$ and $y=0$ from the origin of the coordinates. The thickness of the well-mixed bottom layer is set to $h$. After the elapsed time $t_{0}$, this source of particles is considered distributed following a given profile (exponential or constant) centred along the vertical at $x_{0}$. Then, the particles spread out in the sheared flow. The spatial distribution of the PM concentration from this initial pulse is computed, taking into account the horizontal and vertical advective transport and the horizontal and vertical turbulent mixing.

The coordinate origin is at the seabed, the $x$ - and $y$ axis being horizontal and the $z$-axis vertical. The equation of the transport of the resuspended particles 
Table 1

Notation

\begin{tabular}{|c|c|c|}
\hline Symbol & Definition & Units \\
\hline$C$ & particulate matter concentration & $\mathrm{kg} \mathrm{m}^{-3}$ \\
\hline$C_{0 \max }, C_{0 \max }^{\prime}$ & $\begin{array}{l}\text { maximum particulate matter } \\
(\mathrm{PM}) \text { concentration at } t=t_{0} \text { for } \\
\text { IC } 1 \text { and IC } 2 \text { conditions }\end{array}$ & $\mathrm{kg} \mathrm{m}^{-3}$ \\
\hline$C d_{1}$ & drag coefficient for $z=1 \mathrm{~m}$ & dimensionless \\
\hline$h$ & thickness of the BBL & $\mathrm{m}$ \\
\hline$l_{\mathrm{m}}$ & mixing length & $\mathrm{m}$ \\
\hline$M_{0}$ & $\begin{array}{l}\text { total initial eroded sediment } \\
\text { mass }\end{array}$ & $\mathrm{kg}$ \\
\hline$M$ & total PM cloud mass at time $t$ & $\mathrm{~kg}$ \\
\hline$K_{x}, K_{y}, K_{z}$ & $\begin{array}{l}\text { diffusion coefficients regarding } \\
x-, y \text { - and } z \text {-axis }\end{array}$ & $\mathrm{m}^{2} \mathrm{~s}^{-1}$ \\
\hline$N_{z}$ & turbulent viscosity & $\mathrm{m}^{2} \mathrm{~s}^{-1}$ \\
\hline $\operatorname{Pr}$ & turbulent Prandtl number & dimensionless \\
\hline$T$ & time & $\mathrm{s}$ \\
\hline$U$ & water velocity on $x$-axis & $\mathrm{m} \mathrm{s}^{-1}$ \\
\hline$U_{1}$ & water velocity at $z=1 \mathrm{~m}$ & $\mathrm{~m} \mathrm{~s}^{-1}$ \\
\hline$U_{h}$ & $\begin{array}{l}\text { water velocity at the top of the } \\
\text { BBL }\end{array}$ & $\mathrm{m} \mathrm{s}^{-1}$ \\
\hline$u^{*}$ & friction velocity & $\mathrm{m} \mathrm{s}^{-1}$ \\
\hline$w$ & vertical water velocity & $\mathrm{m} \mathrm{s}^{-1}$ \\
\hline$w_{\mathrm{s}}$ & PM settling velocity & $\mathrm{m} \mathrm{s}^{-1}$ \\
\hline$w_{\text {smin }}$ & minimum PM settling velocity & $\mathrm{m} \mathrm{s}^{-1}$ \\
\hline$x$ & longitudinal axis & $\mathrm{m}$ \\
\hline$y$ & transverse axis & $\mathrm{m}$ \\
\hline$x_{0}$ & origin of the puff injection & $\mathrm{m}$ \\
\hline$z$ & upward vertical axis & $\mathrm{m}$ \\
\hline$z_{10}$ & 10-m altitude & $\mathrm{m}$ \\
\hline$\alpha$ & $u / u_{h}$ power law coefficient & dimensionless \\
\hline$\sigma$ & $\begin{array}{l}\text { horizontal quadratic dispersion of } \\
\text { the initial PM cloud }\end{array}$ & $\mathrm{m}$ \\
\hline$\tau_{\mathrm{b}}$ & instantaneous bottom shear stress & $\mathrm{N} \mathrm{m}^{-2}$ \\
\hline$\left\langle\tau_{\mathrm{b}}\right\rangle$ & mean bottom shear stress & $\mathrm{N} \mathrm{m}^{-2}$ \\
\hline$\tau_{\mathrm{ce}}$ & critical erosion shear stress & $\mathrm{N} \mathrm{m}^{-2}$ \\
\hline$\chi$ & Von Karman constant & dimensionless \\
\hline
\end{tabular}

in $x, y, z$ and $t$ is:

$$
\begin{aligned}
& \frac{\partial C}{\partial t}+\frac{\partial u C}{\partial x}+\frac{\partial\left(\left(w-w_{\mathrm{s}}\right) C\right)}{\partial \mathrm{z}} \\
& =\frac{\partial}{\partial x}\left(K_{x} \frac{\partial C}{\partial x}\right)+\frac{\partial}{\partial y}\left(K_{y} \frac{\partial C}{\partial y}\right)+\frac{\partial}{\partial z}\left(K_{z} \frac{\partial C}{\partial z}\right)
\end{aligned}
$$

where $C$ is the particulate matter concentration, $u$ the horizontal current velocity, $w_{\mathrm{s}}$ the mean settling velocity of the particles, and $K_{x}, K_{y}$ and $K_{z}$ the diffusion coefficients (Table 1).

The vertical water velocity near the bottom must be very small because of the flatness of the bottom regarding the scale of the model. Thus, the vertical convection term can be neglected and the horizontal velocity $u$ can be taken as a function of $z$. The different length scales of vertical and horizontal motions allow us to discard the variation of the horizontal mixing terms. Then Eq. (1) becomes:

$$
\begin{aligned}
& \frac{\partial C}{\partial t}+u(z) \frac{\partial C}{\partial x}+\frac{\partial\left(-w_{\mathrm{s}} C\right)}{\partial z} \\
& =K_{x} \frac{\partial^{2} C}{\partial x^{2}}+K_{y} \frac{\partial^{2} C}{\partial y^{2}}+\frac{\partial}{\partial z}\left(K_{z}(z) \frac{\partial C}{\partial z}\right) .
\end{aligned}
$$

In the lateral direction $y$, the PM transport depends only on the diffusion process. Thus, the PM concentration regarding $y$ can be calculated with a simple Gaussian function. This allows us to split the PM concentration function $C$ into two functions, $f$ and $g$, depending on $(y, t)$ and $(x, z, t)$, respectively, giving:

$C(x, y, z, t)=f(y, t) g(x, z, t)$.

The dimension of $f$ is $l^{-1}$ and that of $g$ is $C \times l$.

Following Fisher (1979), the $f(y, t)$ function is:

$f(y, t)=\frac{1}{\sqrt{4 \pi K_{y} t}} \exp -\frac{y^{2}}{4 K_{y} t}$.

The $g(x, z, t)$ function, in the $(x, z)$ plane can therefore be derived from Eq. (2):

$$
\begin{aligned}
& \frac{\partial g}{\partial t}+u(z) \frac{\partial g}{\partial x}+\frac{\partial\left(-w_{\mathrm{s}} g\right)}{\partial z}-K_{x} \frac{\partial^{2} g}{\partial x^{2}}-\frac{\partial}{\partial z}\left(K_{z}(z) \frac{\partial g}{\partial z}\right) \\
& =0
\end{aligned}
$$

The numerical method used to solve Eq. (4) is described in Appendix A.

\subsection{Water velocity}

The vertical distribution of the horizontal current intensity in the considered bottom layer is expressed according to Csanady (1973):

$u(z)=U_{h}\left(\frac{z}{h}\right)^{\alpha}$

where $h$ is the thickness of the BBL, $U_{h}$ the water velocity at $z=h$ and $\alpha=0.15$ (Fig. 4a). 


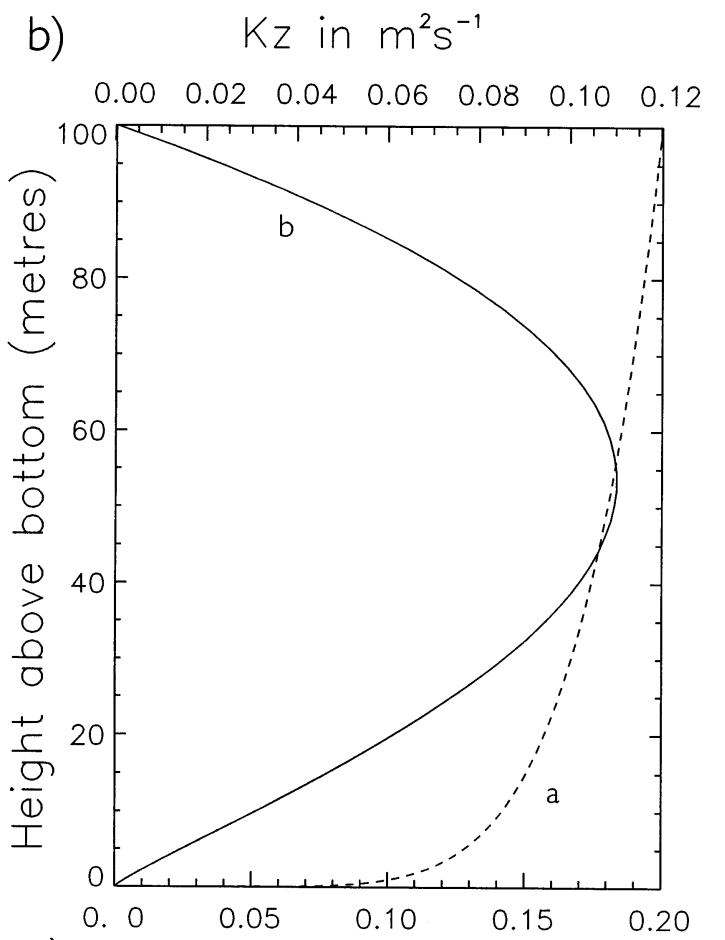

a) Horizontal velocity in $\mathrm{ms}^{-1}$

Fig. 4. Hydrodynamic parameter values in the considered wellmixed 100-m thickness bottom boundary layer.

\subsection{Vertical diffusion coefficient $K_{z}$}

$K_{z}$ is related to the turbulent viscosity $N_{z}$ by:

$K_{z}=\operatorname{Pr} \times N_{z}$

where $\operatorname{Pr}$ is the turbulent Prandtl number (Fig. 4b). According to Lehfeldt and Bloss (1988), for geophysical flows, $\operatorname{Pr}=1$. The turbulent viscosity $N_{z}$ is proportional to a mean representation of the fluctuating velocity and a mixing-length $l_{\mathrm{m}}$ based on the Prandtl hypothesis. Therefore, for a sheared flow with a significant velocity gradient, $N_{z}$ is directly related to the local velocity gradient:

$N_{z}=l_{\mathrm{m}}^{2}\left|\frac{\partial u}{\partial z}\right|$

and the mixing length $l_{\mathrm{m}}$ is expressed as (Boerick and
Hogan, 1977; Sauvaget, 1987):

$l_{\mathrm{m}}=\chi z \sqrt{1-\frac{z}{h}}$

where $\chi=0.4$ is the von Karman constant.

\subsection{Horizontal diffusion coefficients $K_{x}$ and $K_{y}$}

The actual values of the diffusion coefficient $K_{x}$ are difficult to determine. This is because this coefficient is usually discarded in steady state transport equations. The formulae proposed by Fisher (1979) that takes into account the results of Taylor (1954) and Elder (1959), is used here: $K_{x}=5.93 h u^{*}$ and $K_{y}=$ $0.15 h u^{*}$. This method is used only to guide the selection of the $K_{x}$ values and is not actually part of the model. The friction velocity $u^{*}$ depends on the velocity profile and the nature of the sediment. It is determined later following the Soulsby (1983) procedure.

\subsection{Particulate matter settling velocity}

The settling velocity of the particles is calculated in two ways:

- At the starting time of the experiment, the maximum value of the PM concentration $C_{0 \max }$ is considered high enough to allow a settling velocity following the empirical formulae established by Thorn and Parsons (1980):

$$
\begin{aligned}
& w_{\mathrm{s}}=K_{1} C^{n} \quad\left(\text { for } C_{0 \max } \leq 3 \mathrm{~g} \mathrm{l}^{-1}\right) \\
& \text { where } K_{1}=0.513 \times 10^{-3} \text { and } n=1.3 .
\end{aligned}
$$

- At the time when, in a given mesh, $w_{\mathrm{s}}$ decreases and reaches a value of $w_{\text {smin }}$ regarded as a minimum in the considered environment, $w_{\mathrm{s}}$ maintains this $w_{\text {smin }}$. Three specified single $w_{\text {smin }}$ values are used in the model and are discussed later.

\subsection{Boundary conditions}

The value of $K_{z}$ decreases rapidly when the $z$ value tends to $h$ inside the non-stratified water being studied (Fig. 4b). This implies that the upward resuspended PM flux also decreases and becomes very small, following the $K_{z}$ value. Above the bottom-mixed layer, in the stratified water, the values of $K_{z}$ are far less than those inside the BBL. Thus, for this conceptual model, it is assumed that the $K_{z}$ value at 
$z=h$ can be considered null. That implies a null transfer of PM from the considered layer to the water above and then simplify the model computation. The boundary condition at $z=h$ is then:

$\left(K_{z} \frac{\partial C}{\partial z}+w_{\mathrm{s}} C\right)_{z=h}=0$

with $C$ null at the top boundary.

It is assumed that resuspension or erosion processes do not exist at the bottom in the considered domain (excepted for $t \leq t_{0}$ ). Therefore, the bottom altitude can be simply defined by $z=0$. This implies that the particle settling velocity $w_{\mathrm{s}}$ is not modified when the particles reach the bottom (no gradient concentration effect is taken into account near the seabed). That means that the bottom is considered transparent for the PM settling flux. Thus, the bottom boundary condition $(z=0)$ is:

$\Phi_{z=0}=\left(K_{z} \frac{\partial C}{\partial z}+w_{\mathrm{s}} C\right)=w_{\mathrm{s}} C$.

As $K_{z}=0$ at $z=0$, the vertical PM flux on the seabed is only supported by the settling velocity term.

\subsection{Initial conditions}

The initial conditions, concerning the PM concentration in the domain, could affect the shape, with time, of the PM puff. Thus, these initial conditions are carefully studied. At time $t=0$, a single jet of sediment is considered vertically injected in the water, providing at $t=t_{0}$ the initial puff having a total mass $M_{0}$. The spatial distribution of this initial PM puff is assumed to follow a function $C\left(x, y, z, t_{0}\right)$. For physical and numerical reasons, the horizontal distribution cannot be a Dirac, so a Gaussian formulation is used (Fig. 5a):

$$
\begin{aligned}
& C\left(x, y, z, t_{0}\right) \\
& \quad=\frac{1}{\pi \sigma^{2}} \exp \left(-\frac{y^{2}}{\sigma^{2}}\right) \exp \left(-\frac{\left(x-x_{0}\right)^{2}}{\sigma^{2}}\right) e(z)
\end{aligned}
$$

$x_{0}$ is the abscissa of this maximum and $4 K_{y} t_{0}=\sigma^{2}$ is set to 10 to correspond to the very small fraction of the horizontal domain from where the sediment resuspension is supposed to emanate.

There is a real lack of knowledge concerning the shape and the vertical concentration profile of such an

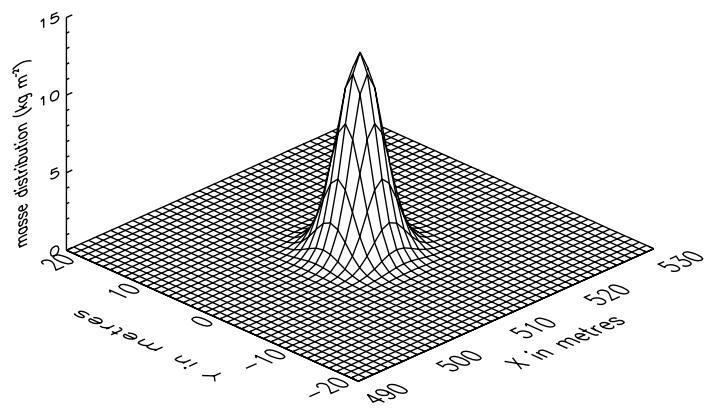

(a)

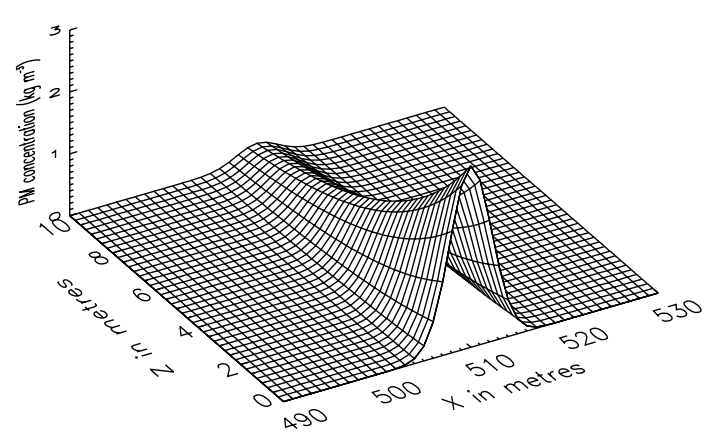

(b) IC-1

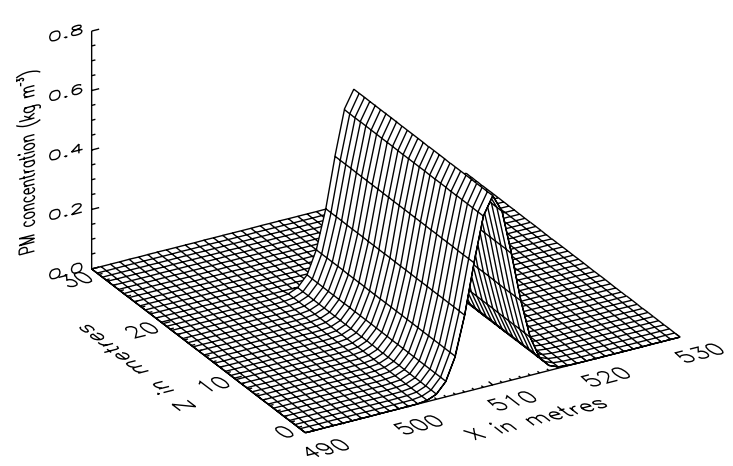

(c) IC-2

Fig. 5. (a) Horizontal repartition of the initial PM mass in the water column above the seabed, as a function of $x$ and $y\left(\mathrm{~kg} \mathrm{~m}^{-2}\right)$. Repartition valid for both the initial conditions IC1 and IC2. (b) and (c) Vertical distribution of the initial PM mass, in the vertical $\left(\mathrm{kg} \mathrm{m}^{-3}\right)$, as a function of $x, z$ and for $y=0$. (b) With an exponential decrease of the PM concentration with height (IC1 conditions). (c) With a constant value of the PM concentration for the first $20 \mathrm{~m}$ above the bottom (IC2 conditions). 
injection of mud coming from the sediment. Estimating a decrease of the PM concentration with the altitude is reasonable, but estimating the rate of this decrease is very difficult. Thus, two different initial vertical distributions of the particulate matter concentration are considered, that provide two expressions of $e(z)$ :

1. An exponential PM concentration decreases, regarding the altitude (Fig. 5b, IC1):

$$
\begin{aligned}
& e(z)=A \exp \left(-\frac{z}{B}\right) \\
& \text { with } \int_{z=0}^{z=h} C(z) \mathrm{d} z=M_{0} \text { and } e_{(z=10)}=\frac{e_{(z=0)}}{10}
\end{aligned}
$$

which leads to:

$$
e(z)=\frac{M_{0}}{z_{10}} \ln (10) \exp \left(-\frac{z}{z_{10}} \ln (10)\right)
$$

where $z_{10}=10 \mathrm{~m}$.

2. A constant PM concentration regarding the altitude $z$ from 0 to $20 \mathrm{~m}$ above the seabed (Fig. 5c, IC2). Thus, $e(z)$ can be simply written as follows:

$$
\begin{aligned}
& e(z)=M_{0} / 20 \text { for } 0<z \leq 20 \mathrm{~m} \quad \text { and } \\
& e(z)=0 \text { for } z>20 \mathrm{~m} .
\end{aligned}
$$

This initial null vertical decreases of $e(z)$ along the first $20 \mathrm{~m}$ above the bottom is non-realistic in nature, but gives a limit case indication of the initial PM concentration effects on the puff shape behaviour.

For both these two initial conditions IC1 and IC2, the initial total PM mass $M_{0}$ of the resuspended sediment is set to the same value.

\section{Actual physical and model parameters values}

The thickness of the well-mixed bottom layer is set to $h=100 \mathrm{~m}$ (Armi and Millard, 1976). Considering the order of magnitude of the bottom geostrophic current in the Gulf of Lions, the current velocity $U_{h}$ at the top of the considered layer is set at first to $U_{h}=$ $0.2 \mathrm{~m} \mathrm{~s}^{-1}$. The values of $0.1-0.8 \mathrm{~m} \mathrm{~s}^{-1}$ are also used. The velocity profile and the related profile of $K_{z}$ are shown in Fig. 4. The $K_{z}$ profile is close to the one obtained by Sauvaget (1982) for equivalent hydro- dynamic conditions; $K_{z}$ is very small above the bottom-mixed layer and can thus be neglected in the model.

The friction velocity $u^{*}$ is determined following Soulsby (1983), taking into account $u$ at $1 \mathrm{~m}$ above the bottom and a drag coefficient $C d$, depending on the nature of the sediment. The relations between $u^{*}$ and the seabed properties, can be written as follows:

$u_{*}^{2}=C d_{1} u_{1}^{2} \quad$ at $1 \mathrm{~m}$ above bottom

where $C d_{1}$ is the drag coefficient value at the altitude $z=1 \mathrm{~m}$. With $U_{h}=0.2 \mathrm{~m} \mathrm{~s}^{-1}$ and using the $C d_{1}$ values reported by Soulsby (1983) for a soft mud sediment (the most probable one), the actual value of $K_{x}$ and $K_{y}$ are, respectively, $1 \mathrm{~m}^{2} \mathrm{~s}^{-1}$ and $0.05 \mathrm{~m}^{2} \mathrm{~s}^{-1}$. For a mud/sand sediment, $K_{x}$ is set to $3 \mathrm{~m}^{2} \mathrm{~s}^{-1}$, which is considered here as a maximum value. To take into account the large uncertainties of the actual main hydrodynamic parameters values, the lowest value of $K_{x}$ is set to $0.1 \mathrm{~m}^{2} \mathrm{~s}^{-1}$. Thus, the model runs with three values of $K_{x}: 0.1,1$ and $3 \mathrm{~m}^{2} \mathrm{~s}^{-1}$.

The computation domain is $2 \mathrm{~km}$ for $x$ and $100 \mathrm{~m}$ for $z$. A rectangular mesh $(1000 \times 51)$ is adopted. The coordinates of the injection point are $x_{0}=551 \mathrm{~m}$ and $y_{0}=0$. The grid size is $2 \mathrm{~m}$ along the $x$-axis. On the vertical axis, there are non-uniform meshes with finer size $(0.5 \mathrm{~m})$ near the seabed. The time step $\Delta t$ is $0.5 \mathrm{~s}$ for the $K_{x}$ values of 0.1 and $1 \mathrm{~m}^{2} \mathrm{~s}^{-1}$ and $0.2 \mathrm{~s}$ for $K_{x}=3 \mathrm{~m}^{2} \mathrm{~s}^{-1}$.

The settling velocity $w_{\mathrm{s}}$ is calculated by using the Thorn formulae (\&3.4), while the PM concentration $C$ is large enough. When, due to the cloud dilution, $w_{\mathrm{s}}$ decreases and reaches the specified $w_{\text {smin }}$ value, $w_{\text {smin }}$ is used; this specified $w_{\text {smin }}$ value is difficult to determine. From the RACACA and PHYCEMED cruises, the $w_{\text {smin }}$ values obtained by applying the Stokes law on a PSD mean size of $12-20 \mu \mathrm{m}$ and a PM true density close to $2 \times 10^{3} \mathrm{~kg} \mathrm{~m}^{-3}$, ends to $10^{-4} \mathrm{~m} \mathrm{~s}^{-1}$. Nevertheless, the Coulter Counter probably underestimates the PSD mean size because the largest probe $(280 \mu \mathrm{m})$ cannot see most of the largest flocs and the particle true density is difficult to evaluate (McCave, 1976; Brun-Cottan, 1986). With a natural environment similar to the ones studied here, $w_{\text {smin }}$ is estimated to be $6 \times 10^{-4} \mathrm{~m} \mathrm{~s}^{-1}$ (Churchill et al., 1994). From various sea and laboratory experiments (Gibbs, 1985; Mehta, 1989; Fennessy et al., 1994; Syvitsky 
et al., 1995; Hill et al., 1998; Manning and Dyer, 1999; Stenberg et al., 1999), the PM mean settling velocity value disperses between $10^{-5}$ and $10^{-3} \mathrm{~m} \mathrm{~s}^{-1}$. Moreover, these determinations were done with steady-state or quasi-steady-state conditions, or the proposed model describes the behaviour of one puff, of which the proportion of large particles is supposed to decrease with time.

Finally, three specified $w_{\text {smin }}$ values are used: $10^{-4}$, $5 \times 10^{-4}$ and $10^{-3} \mathrm{~m} \mathrm{~s}^{-1}$. The model results are fully discussed and illustrated for $w_{\text {smin }}=5 \times 10^{-4} \mathrm{~m} \mathrm{~s}^{-1}$. For the $10^{-4}$ and $10^{-3} \mathrm{~m} \mathrm{~s}^{-1}$ values, considered as boundaries esteems, only synthesised results are shown.

For the initial conditions IC1 (exponential profile), the total mass $M_{0}$, injected into the water column is determined by setting the maximum initial PM to $C_{0 \max }=3 \mathrm{~g} \mathrm{l}^{-1}$ and the scale of the area of resuspension to $10 \mathrm{~m}^{2}\left(2 \sigma^{2}\right.$ of $\left.M_{0}\right)$. This gives $M_{0}=400 \mathrm{~kg}$. From $M_{0}$ determined for the IC1 conditions, the $C_{0 \text { max }}^{\prime}$ value for the initial conditions IC2 is $0.64 \mathrm{~g} \mathrm{l}^{-1}$.

The background PM concentration is not taken into account, so the reported PM values $C$ calculated by the model are the excess PM concentration, regarding the background value. The model operates from 0 to $10^{4} \mathrm{~s}$.

\section{Results and discussion}

The numerical integration of the PM mass in the computation domain, plus the mass lost by sedimentation through the bottom boundary, is very close to $M_{0}$ up to $t=4 \times 10^{4} \mathrm{~s}$. That means that the PM mass conservation is maintained.

The minimum reasonable PM concentration to be detected in the seawater, with the actual techniques in use is considered $30 \mu \mathrm{g} 1^{-1}$. This PM value represents, in this model, the minimum detectable excess PM concentration originating from the resuspended cloud. The $\log$ of this relative value, considering $C_{0 \max }=3 \mathrm{~g}^{-1}$, is then: $\log \left[\left(30 \times 10^{-6} \mathrm{~g} \mathrm{l}^{-1}\right) /\left(3 \mathrm{~g} \mathrm{l}^{-1}\right)\right]=-5$.

\subsection{Main conditions}

For $U_{h}=0.2 \mathrm{~m} \mathrm{~s}^{-1}$ and $w_{\text {smin }}=5 \times 10^{-4} \mathrm{~m} \mathrm{~s}^{-1}$, the longitudinal sections in $x, z$ (Figs. 6 and 7) represent the spatial distribution of the excess PM concentration, 500 and $5000 \mathrm{~s}$ after the injection time. These sections are drawn for $y=0$. Quickly after the injec- tion time (500 s), the influence of the initial conditions IC2, compared with those of the IC1, can be neglected on the PM cloud size, but not on the vertical PM profiles (Figs. 8 and 9). The intensity of the horizontal mixing influences the size of the resuspended cloud, mainly during the first $1000 \mathrm{~s}$ after the injection time. After $5000 \mathrm{~s}$, the size and the shape of the PM cloud are almost the same for the $K_{x}$ values of 1 and $3 \mathrm{~m}^{2} \mathrm{~s}^{-1}$. The cloud shape corresponding to $K_{x}=0.1 \mathrm{~m}^{2} \mathrm{~s}^{-1}$, shows the effect of a very small mixing rate.

The cloud expansion implies an increase of the PM concentration around the injection area. Thus, the volume in the seawater, contained inside a closed surface area defined by a given PM concentration value, expands at first. However, after some time, depending on the previous value, this volume contracts. For instance, the isoline -5 , which represents the intersection of the surface -5 and the $y=0$ vertical plane, expands continuously (Figs. 6 and 7). At $5000 \mathrm{~s}$, it reaches a horizontal size of $800 \mathrm{~m}$ long near the seabed and $500 \mathrm{~m}$ at the altitude of $40 \mathrm{~m}$. After $t \sim 6000 \mathrm{~s}$ the isoline -5 retracts to the seabed and disappears at $t \sim 10^{4} \mathrm{~s}$. However, during this lapse of time, the isolines -2 and -3 (the highest PM concentration) are clearly perceivable at $500 \mathrm{~s}$ but almost completely disappear after $5000 \mathrm{~s}$.

That means that the initial PM cloud is no longer detectable, with the actual model parameters values, after a very few hours following the injection time.

PM concentration profiles at 500 and $5000 \mathrm{~s}$, at a given distance $x$ from the injection origin, are shown in Figs. 8 and 9; these profiles are expressed in the non-dimensional form $\left(C(x, y=0, z, t) / C_{0 \max }\right)$. Some of these profiles exhibit a maximum PM concentration above the seabed (bump). Depending on $K_{x}$ and $x$, both Rouse and bump profiles appear. The lower the $K_{x}$ value is, the more amplified these bumps are. For a given time after the injection, the presence of the bump is very sensitive to the distance $x$ (from the injection origin) of the profile.

\subsection{Bound conditions}

The model sensitivity is tested for some $U_{h}$ and $w_{\text {smin }}$ parameter values. There, the only initial condition considered is IC1. 

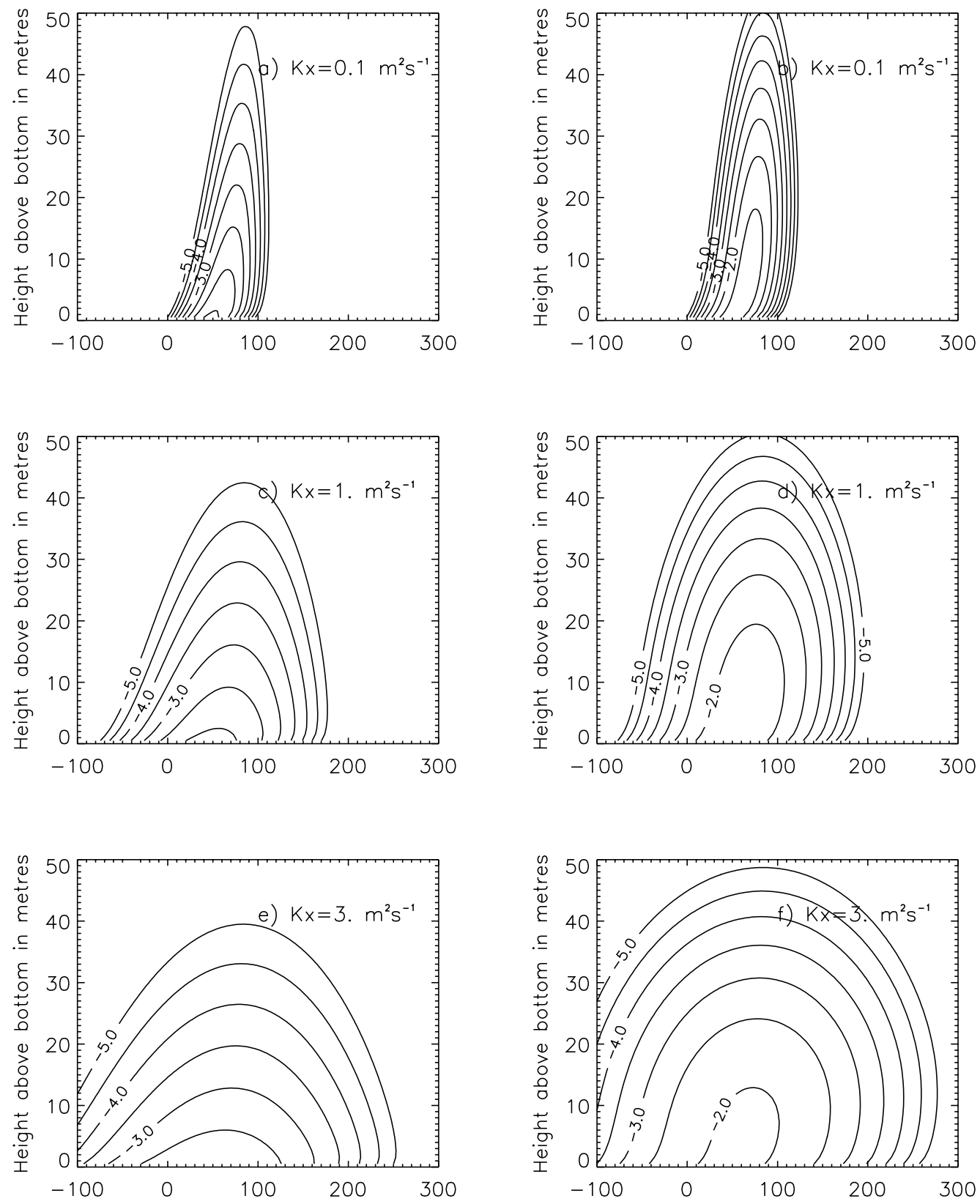

Distance from the injection point in metres Initial Conditions $1:(a, c$ and $e)$

Distance from the injection point in metres Initial Conditions 2 : (b,d and f)

Fig. 6. Longitudinal sections showing the excess PM concentration isolines $\log \left(C_{(G)} / C_{0 \max }\right) 500 \mathrm{~s}$ after the injection time $(y=0)$. The left and right columns show the shape of the PM cloud for the initial conditions IC1 and IC2. 

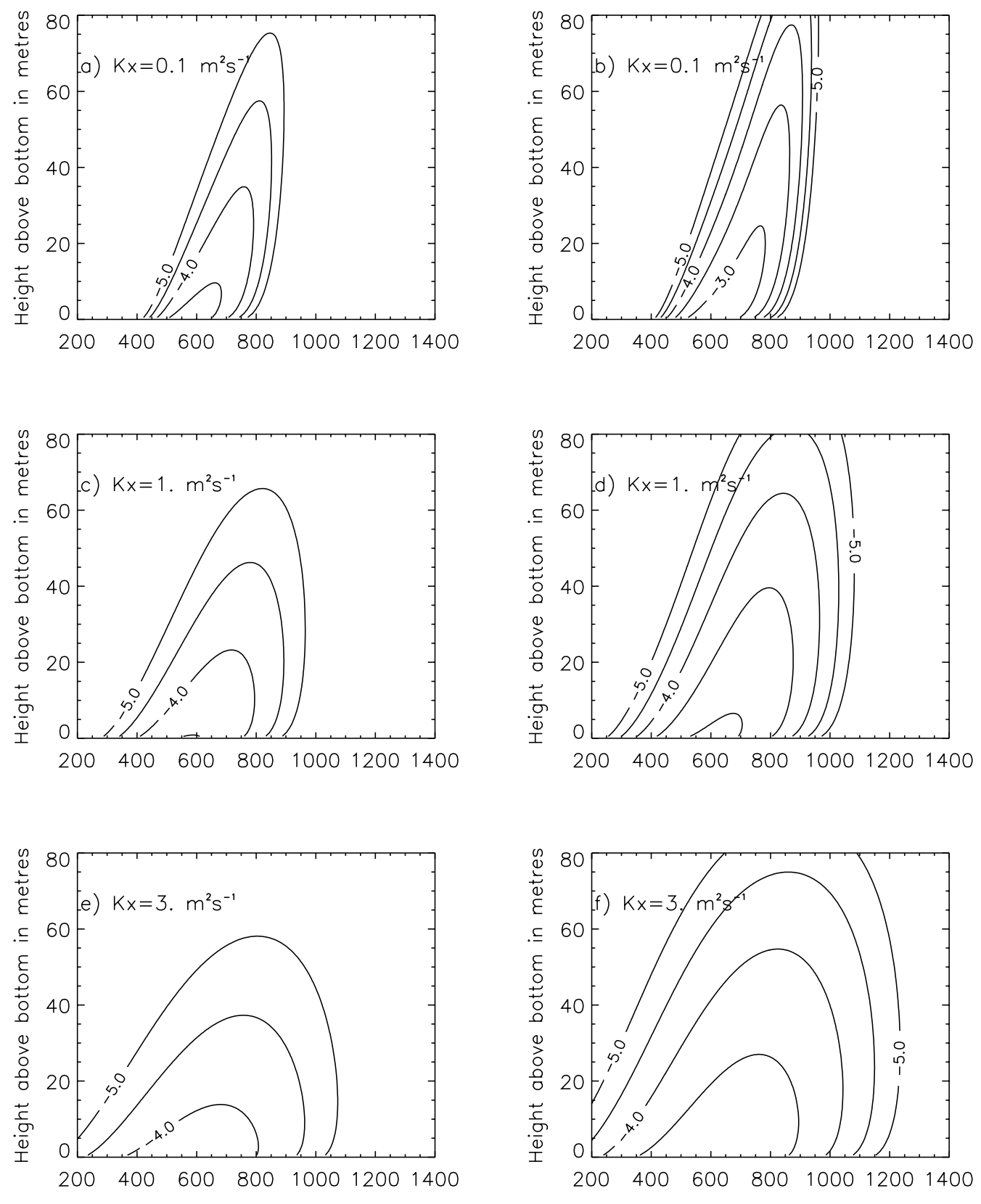

Distance from the injection point in metres

Initial Conditions 1 : (a,c and e)

Distance from the injection point in metres

Initial Conditions $2:(b, d$ and $f)$

Fig. 7. Longitudinal sections showing the excess PM concentration isolines $\log \left(C_{(G)} / C_{0 \max }\right) 5000 \mathrm{~s}$ after the injection time $(y=0)$. The left and right columns show the shape of the PM cloud for the initial conditions IC1 and IC2. 

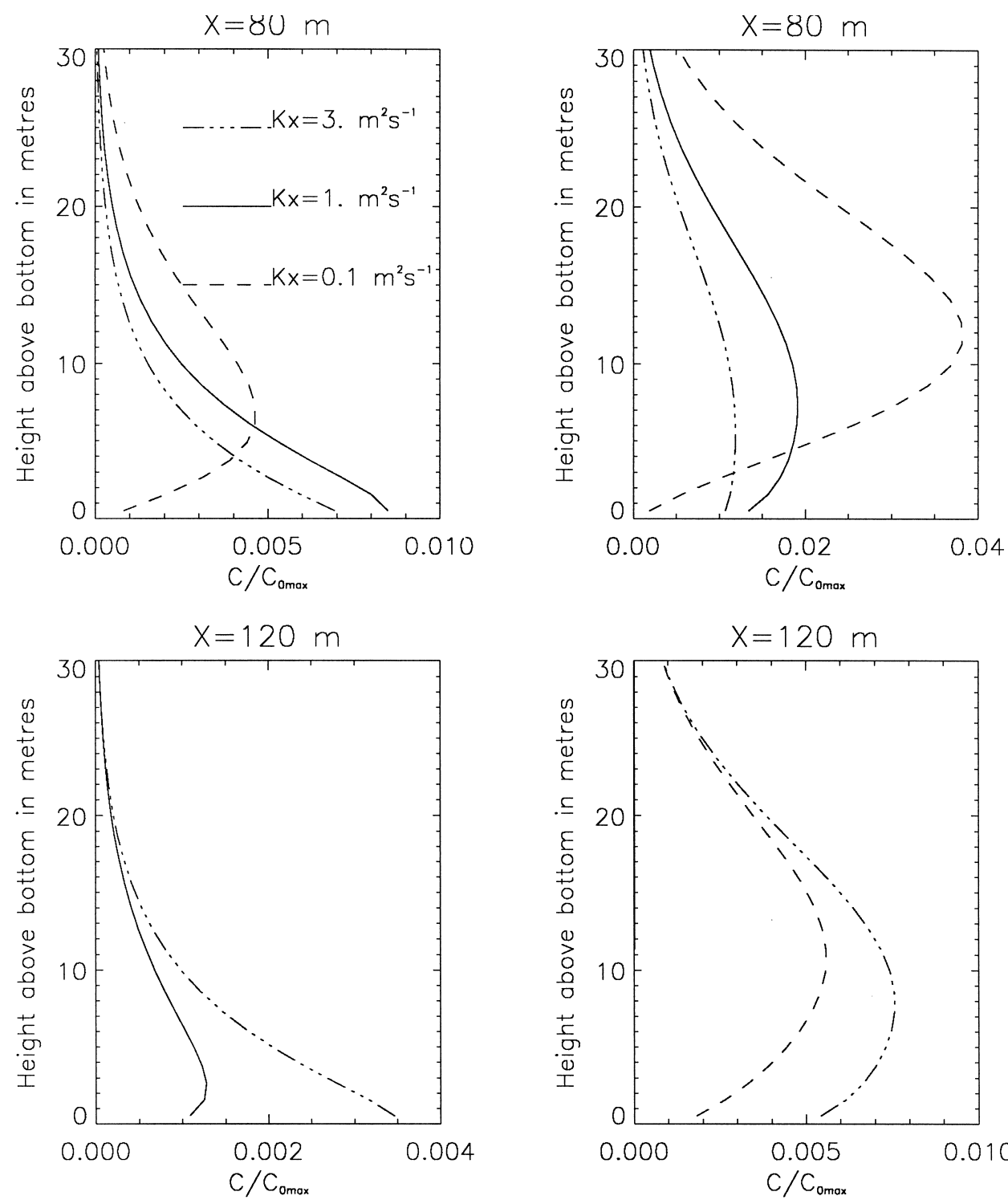

Initial Conditions 1

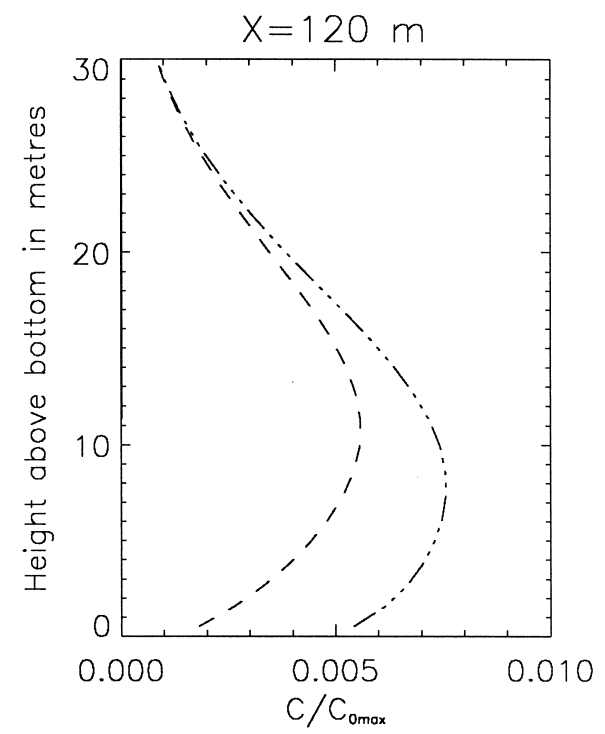

Initial Conditions 2

Fig. 8. Excess PM concentration profiles regarding two distances $x$ from the injection point $(y=0) 500 \mathrm{~s}$ after the injection time, with the three considered values of $K_{x}$. The left and right columns are related to the initial conditions IC1 and IC2.

The model sensitivity for $U_{h}$ is analysed with $w_{\text {smin }}=5 \times 10^{-4} \mathrm{~m} \mathrm{~s}^{-1}, K_{x}$ and $K_{y}$ are recalculated complying with $U_{h}$. The results are shown in Fig. 10 for the $0.1-0.8 \mathrm{~m} \mathrm{~s}^{-1} U_{h}$ values. As it could be expected, the size of the PM cloud expands highly when $U_{h}$ increases.
To show the behaviour of the total puff (in its entirety), depending on $U_{h}$, the altitude and the PM concentration of the gravity centre $G$ of the PM cloud are introduced, regarding the time after the PM injection. The influence of $U_{h}$ on the vertical location of $G$ is shown in Fig. 11b. The altitude of $G$ continuously 

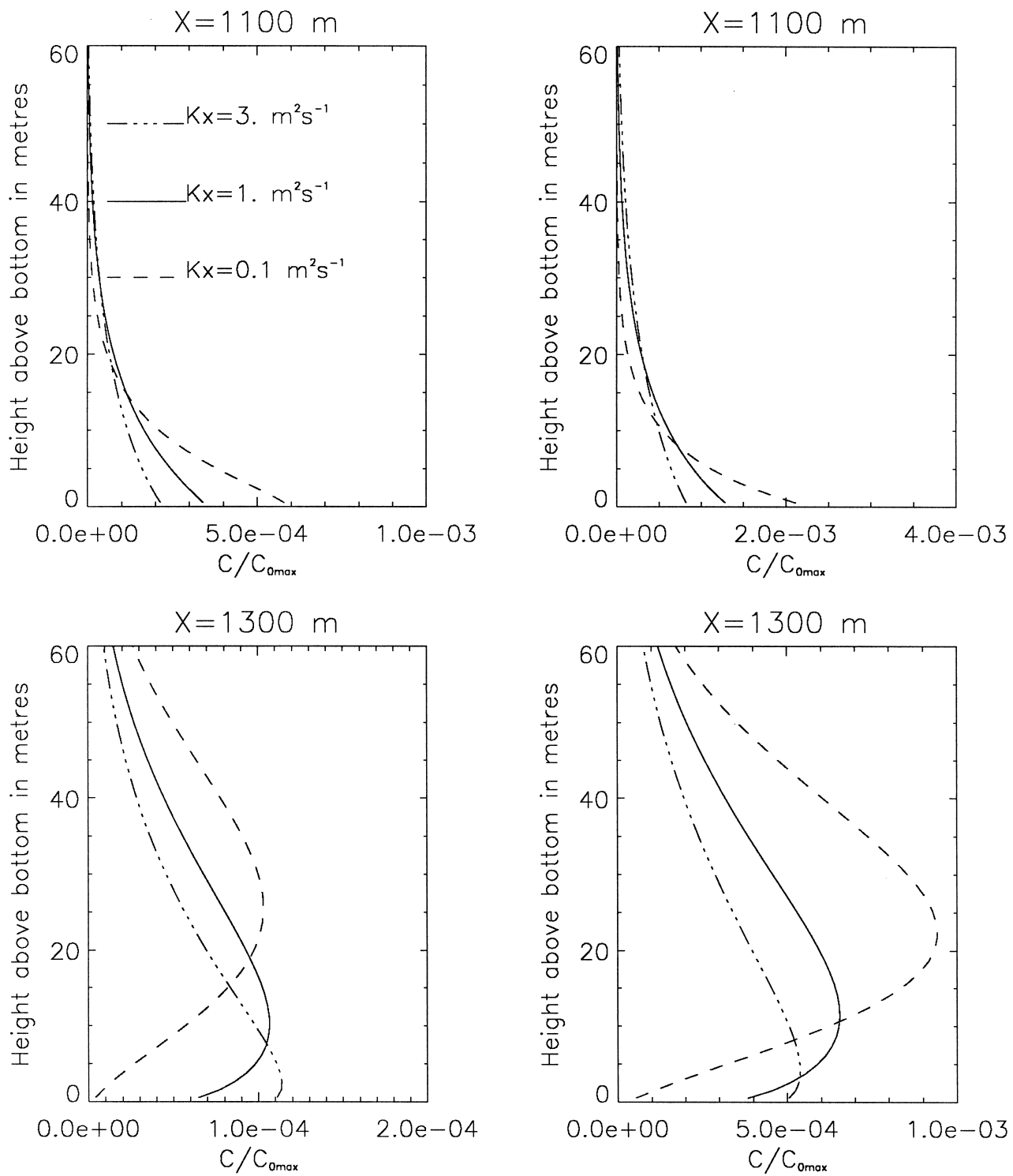

Initial Conditions 1

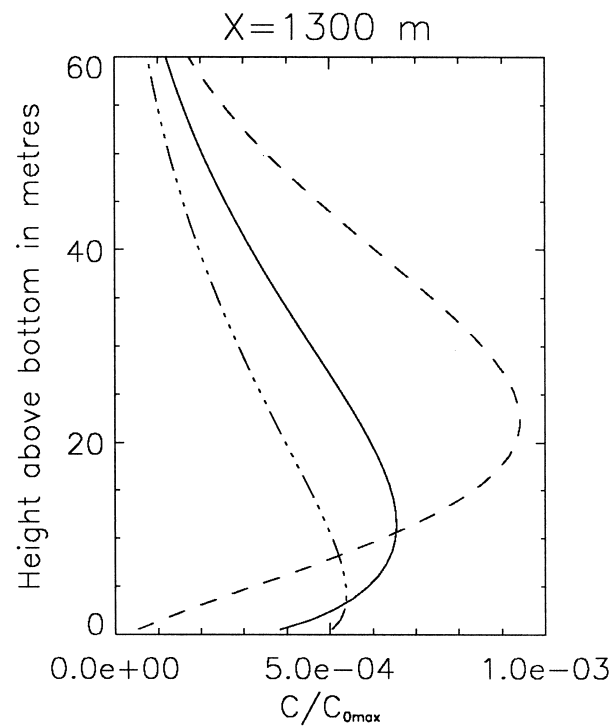

Initial Conditions 2

Fig. 9. Excess PM concentration profiles regarding two distances $x$ from the injection point $(y=0) 5000 \mathrm{~s}$ after the injection time, with the three considered values of $K_{x}$. The left and right columns are related to the initial conditions IC1 and IC2.

increases and strongly depends on the $U_{h}$ value. This altitude does not in fact, exceed $60 \mathrm{~m}$ for the highest value of $U_{h}=1 \mathrm{~m} \mathrm{~s}^{-1}$. The $U_{h}$ value does not affect the decrease of the PM concentration at $G$ as much (Fig. 11b).

The model sensitivity to the minimum PM settling velocity $w_{\text {smin }}$ is analysed for the two specified values, $10^{-4}$ and $10^{-3} \mathrm{~m} \mathrm{~s}^{-1}$, compared with the central one of $5 \times 10^{-4} \mathrm{~m} \mathrm{~s}^{-1}$. This at $t=$ $5000 \mathrm{~s}$, with $U_{h}=0.2 \mathrm{~m} \mathrm{~s}^{-1}$ and $K_{x}=1 \mathrm{~m}^{2} \mathrm{~s}^{-1}$. The longitudinal sections in $x, z$ (Fig. 12) show that $w_{\text {smin }}$ has a very minor influence on the 


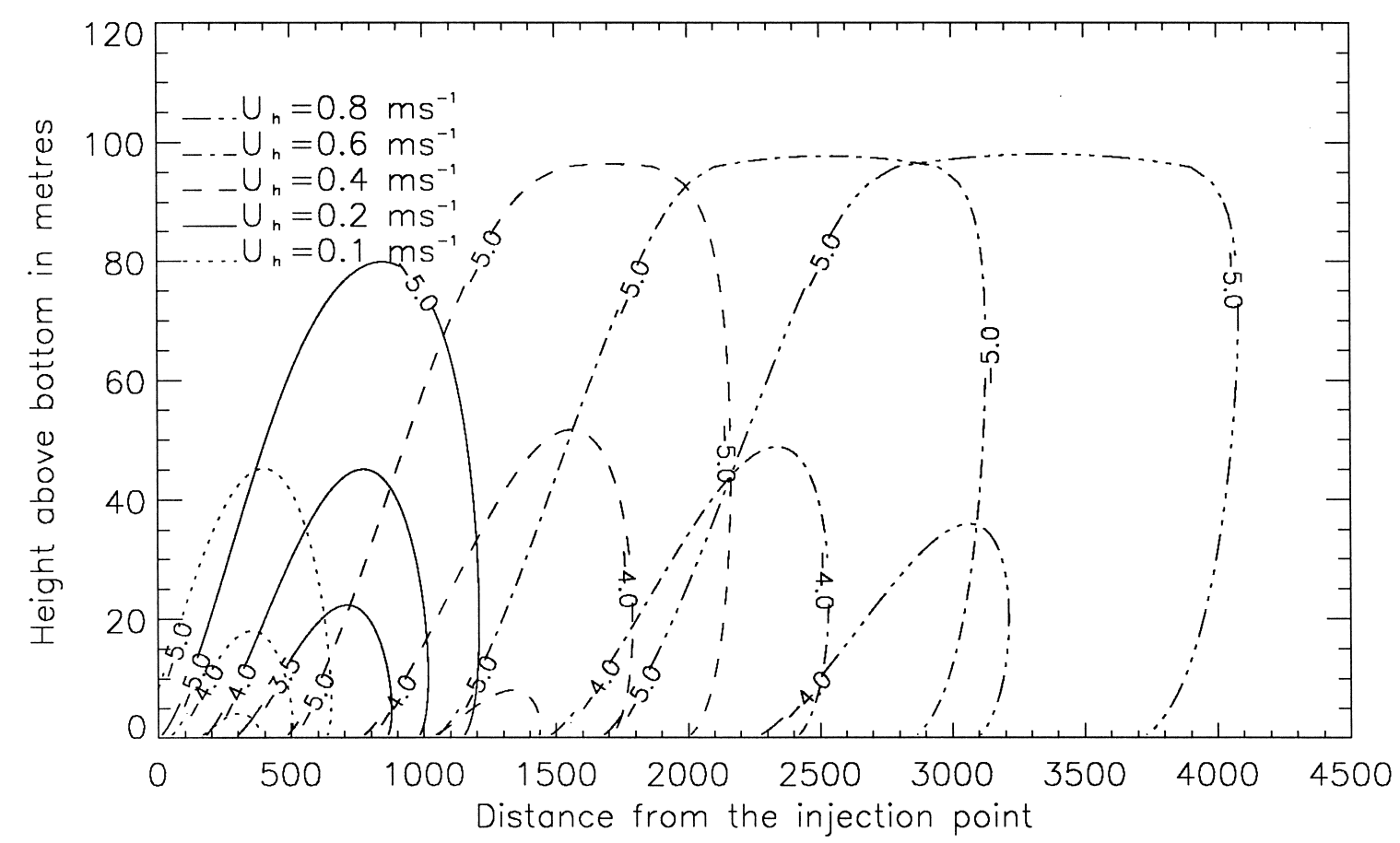

Fig. 10. Longitudinal sections showing the excess PM concentration isolines $\log \left(C_{(G)} / C_{0 \max }\right) 5000 \mathrm{~s}$ after the injection time $(y=0)$, regarding the water velocity $U_{h}$ at the top of the BBL. With $w_{\text {smin }}=5 \times 10^{-4} \mathrm{~m} \mathrm{~s}^{-1}$.

size of the PM cloud, mainly affected by the turbulent mixing. The influence of $w_{\text {smin }}$ is more perceptible on the PM concentration profiles (Fig. 13), but the changes on the profile's shape are not meaningful.

The fraction of $M_{0}$ lost by sedimentation on the seabed, regarding time, rely on $w_{\text {smin }}$ and is quasiunconstrained by $K_{x}$ (Fig. 14).

\subsection{Relation between the model and the observed results}

When bump profiles are obtained by the model, their shape is similar to the one observed for the PM mass, or size signal, obtained during the RACACA, PHYCEMED and CYBELE cruises. The bump profiles show a maximum of the PM concentration or mean size values at an altitude of around $10 \mathrm{~m}$ for the PHYCEMED data and $20 \mathrm{~m}$ for the RACACA ones; the CYBELE cruise results are not considered in this part of the discussion because they are obtained on the shelf break, at some shallower depths, where fluctuating lateral transport could plausibly apply. Considering the model parameters that means that the casts were done during the first half an hour after the injection time for the PHYCEMED example and within the first $2 \mathrm{~h}$ for the RACACA ones. No more comparisons can be made concerning the PM concentration because the total lack of data concerning the initial mass $M_{0}$ and the initial conditions of resuspended sediment in nature.

After some thousands of seconds after the injection time, the size of the area where bump profiles could appear is small, considering the size of the simulation domain. Thus, the probability of finding a bump profile in the considered domain, using the model or the measurements, is small.

From these results, we can suggest that the particulate material above the seabed may originate from a continuous addition of resuspended sediment impulses. These sediment impulses and their associated clouds could appear each time the instantaneous bottom shear stress $\tau_{\mathrm{b}}$, induced by the water friction, exceeds locally and for a short duration the 

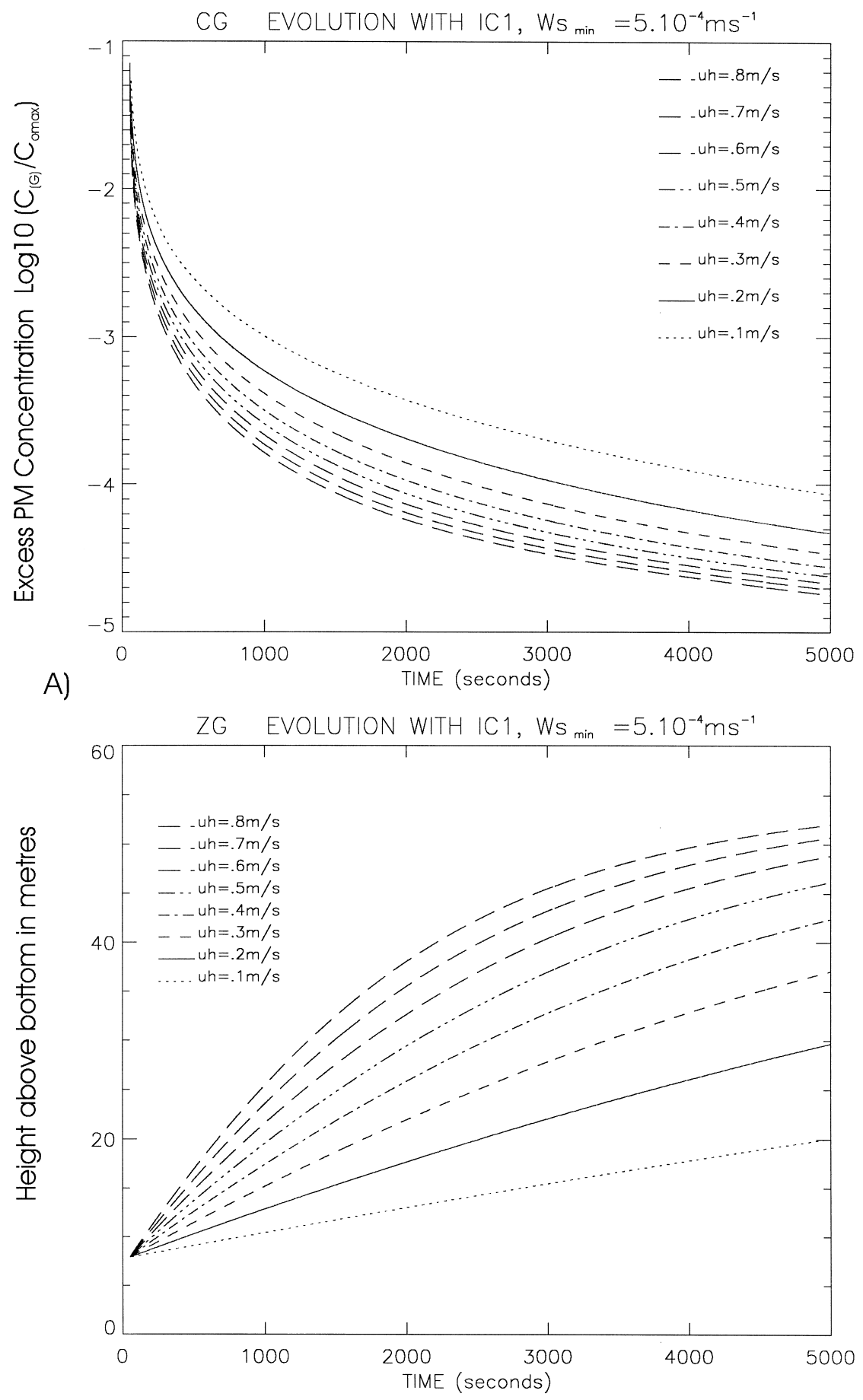

B)

Fig. 11. (A) and (B) Concentration and altitude characteristics of the gravity centre $G$ of the PM cloud, as a function of time. (A) and (B) are related to the IC1 conditions, with $K_{x}=1 \mathrm{~m}^{2} \mathrm{~s}^{-1}$ and $w_{\text {smin }}=5 \times 10^{-4} \mathrm{~m} \mathrm{~s}^{-1}$. (A) Decreases with time of the mass concentration $C_{(G)}$ at $G$,: $\log \left(C_{(G)} / C_{0 \max }\right)$. (B) Variation with time of the altitude of $G$. 


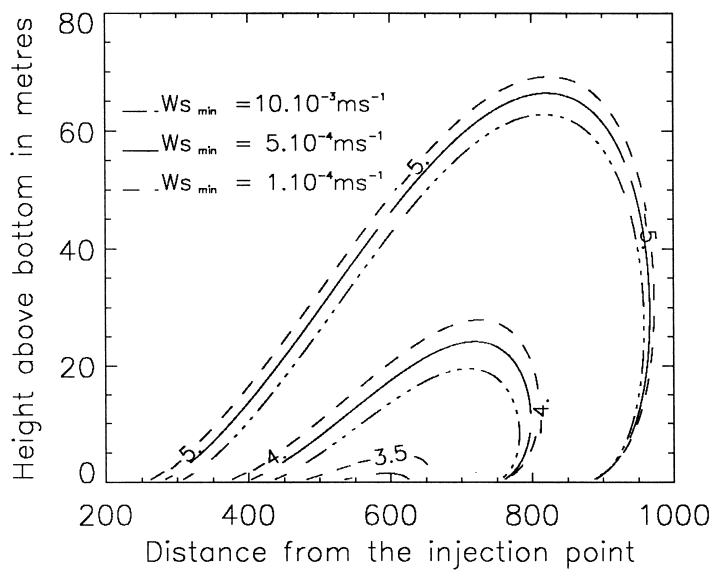

Fig. 12. Longitudinal sections showing the excess PM concentration isolines $\log \left(C_{(G)} / C_{0 \max }\right) 5000 \mathrm{~s}$ after the injection time $(y=0)$, related to the IC1 conditions, with $K_{x}=1 \mathrm{~m}^{2} \mathrm{~s}^{-1}$ and for three values of $w_{\text {smin }}$.

critical erosion shear stress $\tau_{\text {ce }}$ of the sediment. This means that the resuspension process can occur with a mean $\left\langle\tau_{\mathrm{b}}\right\rangle$ value $\left(\left\langle\tau_{\mathrm{b}}\right\rangle<\tau_{\mathrm{b}}\right.$ maximum) less than $\tau_{\mathrm{ce}}$ $\left(\left\langle\tau_{\mathrm{b}}\right\rangle<\tau_{\mathrm{ce}}\right)$, but temporarily with an instantaneous $\tau_{\mathrm{b}}>\tau_{\mathrm{ce}}$ status induced by the mean current velocity, less than $\tau_{\text {ce. }}$. These impulses can happen following a random procedure, in space and time; from the model, they are identifiable, regarding the background PM concentration, only during a few hours.

Many processes can exist which induce an increase in the mean $\left\langle\tau_{\mathrm{b}}\right\rangle$ value, locally and temporarily. These could be surface waves in shallow waters or, more generally, internal waves in deeper waters; energy transfer from strong wind could be also a good possible origin. The wind was calm to moderate (force 2-5) during the RACACA cruise. During the PHYCEMED cruise, the wind was calm except through a 3-day period (force 8-9), 2 days before the cast Gy-LB showing the bump profile (Fig. 1). The profile (Fig. 2) shown during the CYBELE cruise was obtained throughout a force 10 storm following a force 11 (no data were collected during the force 11). Thus, wind energy could be invoked for the bump profiles obtained during the PHYCEMED and CYBELE cruises, but not for the RACACA one.

Coupled measurements of PM concentration and current velocity where obtained during the HEBBLE programme (Hollister et al., 1984), in an area centred $730 \mathrm{~km}$ East of Boston. The results obtained with a
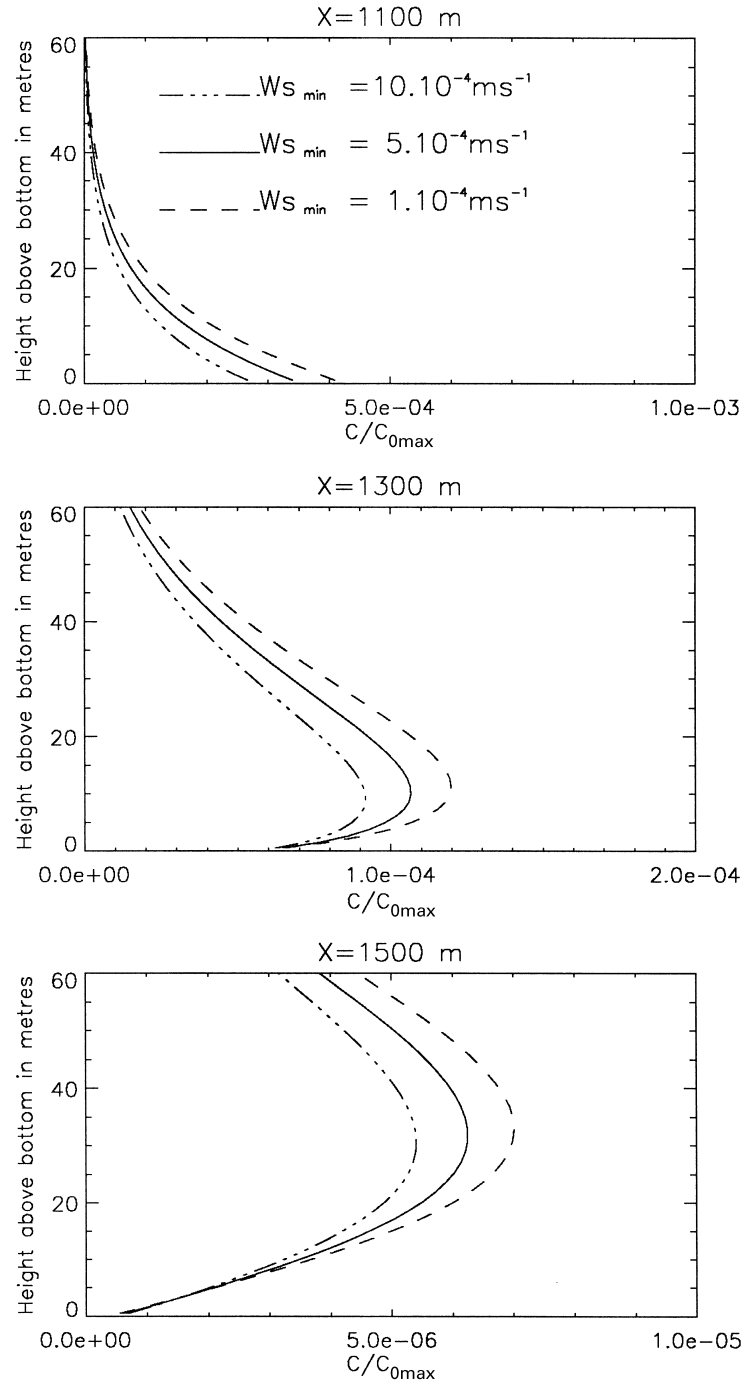

Fig. 13. Excess PM concentration profiles for three distances $x$ from the injection point $(y=0) 5000 \mathrm{~s}$ after the injection time, related to the IC1 conditions, with $K_{x}=1 \mathrm{~m}^{2} \mathrm{~s}^{-1}$ and for three values of $w_{\text {smin }}$.

tripod moored at $4800 \mathrm{~m}$ depth, show rapid fluctuations of the PM concentration few metres above the seabed, by sometimes a factor of 100 regarding the background level. These fluctuations are strongly tied with bottom current velocity maxima and coincide with severe storms periods. The size of the spatially confined sediment resuspension area must be incomparably less than the storm one. Even if a storm concerns a broad area, the energy transmitted down 


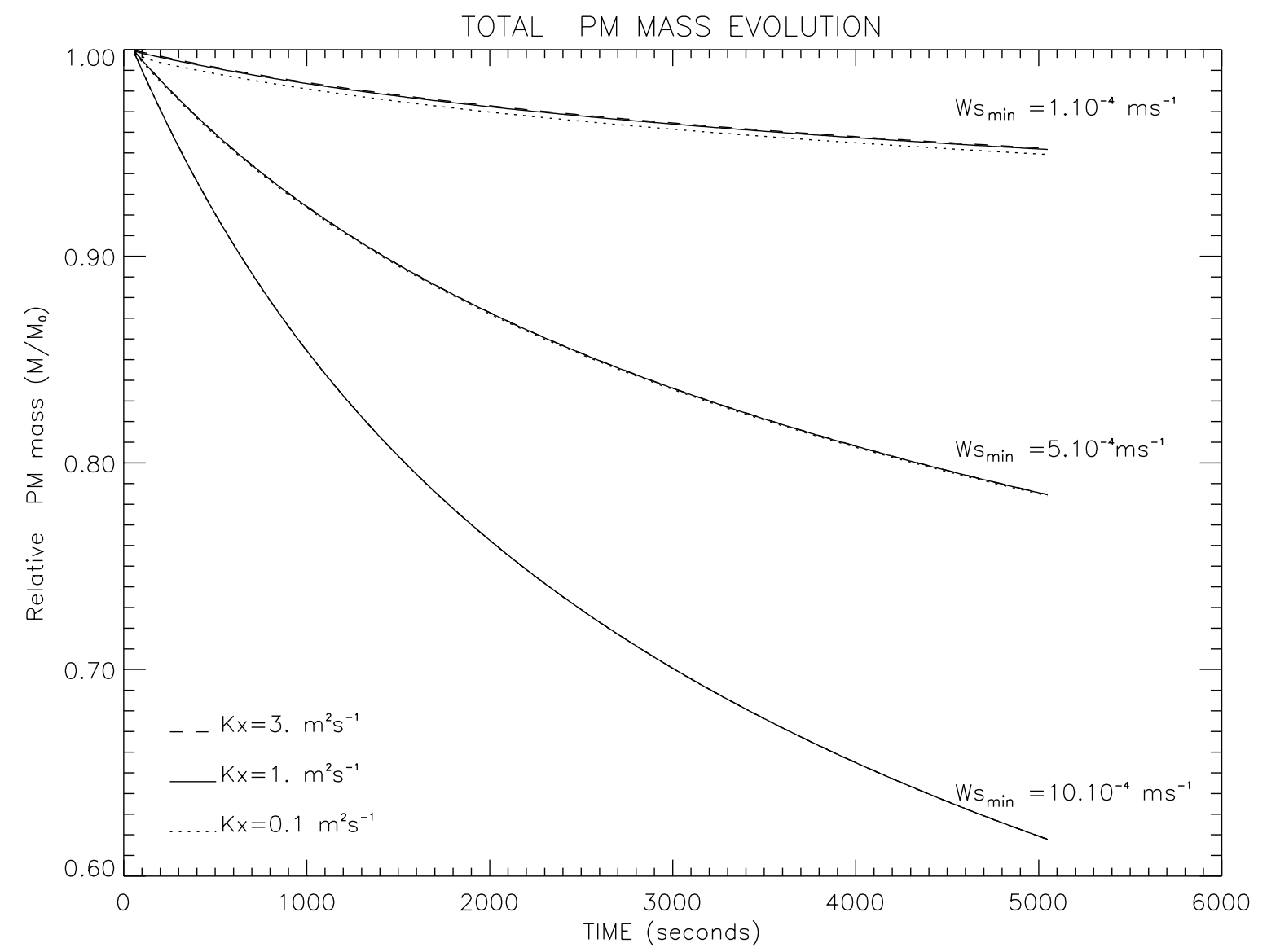

Fig. 14. Decreases with time of the mass ration $M / M_{0}$ originating from the initial resuspended sediment pulse $M_{0}$, related to the IC1 conditions and for three values of $K_{x}$ and of the minimum assumed PM settling velocity $w_{\mathrm{s}}$. The PM mass lost is due to the settling of the suspended particles throughout the bottom boundary.

to the deep is dispersed by eddies having size far less than the storm area one, sizes scattered along the Kolmogorov scale (including bursts).

The trawling from fishing boats is also mentioned under certain circumstances (Churchill et al., 1994). A convincing argument comes from the data obtained during the SEEP-II experiment, showing the beam attenuation 3 mab and the bottom shear stress as a function of time (Churchill et al., 1994). These data show that time variability and beam attenuation are not correlated with the calculated bottom shear stress. This matches the transportation of sediment impulses originating from areas where an intense positive variation of $\left.\left\langle\tau_{\mathrm{b}}\right\rangle\left(\tau_{\mathrm{b}}\right\rangle\left\langle\tau_{\mathrm{b}}\right\rangle\right)$ can occur when, simultaneously, the bottom shear stress has only its mean value at the deployment location.

\section{Conclusions}

A simplified 3-D model is proposed to simulate the spatial distribution of the particles originating from an injected puff of cohesive sediment in a sheared flow with turbulent diffusion, as could be the case in the Bottom Boundary Layer. The time when the clouds resulting from that kind of puff becomes detectable, following the model concept 
and with the considered initial conditions, is merely a few hours.

Numerical casts done in the simulated cloud issued from this sediment injection show exponential profiles and bump profiles. The bump profiles appear only at some given distances from the injection point. They have a shape similar to the ones found during scientific cruises in the North Mediterranean and in the New York bight.

The development of the bump profiles is strongly affected by the intensity of the horizontal convection and the vertical diffusion.

The mechanisms involved explaining these results signify that the mean bottom shear stress value necessary to trigger the erosion process can be far less than the critical sediment erosion shear stress. This suggests that the sediment resuspension flux could be, in many circumstances, more important than the one shown by the standard estimated method.

The principle of erosion and transportation of sediment originating from many dispersed impulses match the time variability of the particulate matter concentration often encountered above the seabed.

\section{Acknowledgements}

This work is supported by the Commission of the Programmes of the European Community under the contract MAST.CT.90.0063.DTTE.

\section{Appendix A}

\section{A.1. Numerical method}

A rectangular mesh is used for the $x, y$ dimensions. A cell centred position is adopted for the unknown variables.

A semi-implicit Cranck Nicholson scheme is used to calculate the vertical motion, while an explicit scheme is employed to calculate the horizontal advection. For each time step, a three-diagonal system is obtained and the Von Neuman procedure guarantees the stability of the horizontal scheme with the space and time parameters used.
The discrete form of Eq. (2) is then:

$$
\begin{aligned}
& \frac{g_{i, j}^{n+1}-g_{i, j}^{n}}{\Delta t}+\frac{u_{j}^{n}}{\Delta x}\left[\left(g_{i, j}^{n}-g_{i-1, j}^{n}\right)\right. \\
& \left.+\frac{1}{2} \psi_{i-1 / 2}^{+}\left(g_{i, j}^{n}-g_{i-1, j}^{n}\right)-\frac{1}{2} \psi_{i-3 / 2}^{+}\left(g_{i-1, j}^{n}-g_{i-2, j}^{n}\right)\right] \\
& -\frac{1}{2 \Delta z_{j}}\left[\left(W s_{i, j+1 / 2} g_{i, j+1}-W s_{i, j-1 / 2} g_{i, j}\right)\right. \\
& \left.+K z_{j+1 / 2} \frac{\left(g_{i, j+1}-g_{i, j}\right)}{0.5\left(\Delta z_{j}+\Delta z_{j+1}\right)}-K z_{j-1 / 2} \frac{\left(g_{i, j}-g_{i, j-1}\right)}{0.5\left(\Delta z_{j-1}+\Delta z\right)}\right]^{n+1} \\
& =\frac{1}{2 \Delta z_{j}}\left[\left(W s_{i, j+1 / 2} g_{i, j+1}-W s_{i, j-1 / 2} g_{i, j}\right)\right. \\
& \left.+K z_{j+1 / 2} \frac{\left(g_{i, j+1}-g_{i, j}\right)}{0.5\left(\Delta z_{j}+\Delta z_{j+1}\right)}-K z_{j-1 / 2} \frac{\left(g_{i, j}-g_{i, j-1}\right)}{0.5\left(\Delta z_{j-1}+\Delta z\right)}\right]^{n} .
\end{aligned}
$$

The discrete form of the vertical motion is classical (central differences in space), but the second term of Eq. (A1), which represents the horizontal motion, needs some explanations. To reduce the numerical diffusion of a single first-order upwind scheme and to avoid the numerical oscillations of a high-resolution scheme, the second-order TVD semi-discretized scheme is used (Hirsch, 1992). For this last scheme, a non-linear limiting function $\Psi^{+}$restricts the variability of the horizontal $g$-gradient values. The limiter $\Psi^{+}$is an operator that allows us to move continuously from a second-order upwind scheme to a first-order upwind scheme when discontinuities appear.

The limiter $\Psi$ is defined as follows:

$\Psi_{i-1 / 2}^{+}=\Psi\left(r_{i-1 / 2}^{+}\right) \quad$ with $r_{i-1 / 2}=\frac{g_{i+1, j}-g_{i, j}}{g_{i, j}-g_{i-1, j}}$

The limiter minmod is used in this application and is written as:

$$
\begin{aligned}
\Psi(r) & =\operatorname{minmod}(r, 1) \\
& =\operatorname{Sign}(r) \max [o, \min (|r|, \operatorname{Sign}(r))]
\end{aligned}
$$

The values of this function range from 0 when $r$ is negative to 1 when $(r \geq 1)$. 


\section{References}

Armi, L., Millard, R., 1976. The bottom boundary layer of the deep ocean. J. Geophys. Res. 81 (27), 83-111.

Biscaye, P.E., Eittreim, S.L., 1977. Suspended particulate loads and transports in the nepheloid layer of the abyssal Atlantic Ocean. Mar. Geol. 23, 155-172.

Boerick, R.R., Hogan, J.M., 1977. An x-z hydraulic/thermal model for estuaries. Hyd. Div. ASCE 103, 19-37.

Brun-Cottan, J.C., 1984. Remise en suspension des particules à l'interface eau-sédiment en Méditerranée Occidentale. Campagne PHYCEMED 83. VII Journées Etud. Pollutions, Lucerne, CIESM.

Brun-Cottan, J.C., 1986. Vertical transport of particles within the Ocean. NATO-ASI, The Role of Air Sea Exchange in Geochemical Cycling. Reidel, Dordrecht, pp. 83-111.

Churchill, J.H., Wirick, C.D., Flagg, C.N., Pietrafesa, L.J., 1994. Sediment resuspension over the continental shelf east of the Delmarva Peninsula. Topical studies in oceanography: shelf edge exchange processes in the Southern Middle Atlantic Bight: SEEP-II. Deep-Sea Res. 41, 341-363.

Csanady, G.T., 1973. Turbulent Diffusion in the Environment, Geophysics and Astrophysics Monograph, Reidel, Dordrecht.

Elder, J.W., 1959. The dispersion of marked fluid in turbulent shear flow. J. Fluid Mech. 5, 544-560.

Fennessy, M.J., Dyer, K.R., Huntley, D.A., 1994. INSSEV: an instrument to measure the size and settling velocity of flocs in situ. Mar. Geol. 117, 107-117.

Fisher, H., 1979. Mixing in Inland and Coastal Waters, Academic Press, New York.

Gibbs, R., 1985. Estuarine flocs: their size, settling velocity and density. J. Geophys. Res. 90 (C2), 3249-3251.

Hill, P.S., Syvitski, J.P., Cowan, E., Powell, R.D., 1998. In situ observations of flocs settling velocities in Glacier Bay, Alaska. Mar. Geol. 145, 85-94.

Hirsch, C., 1992. Numerical Computation of Internal and External Flows, vol. 2, Wiley, New York.
Hollister, D., Nowell, A.R.M., Jumars, P.A., 1984. The dynamic abyss; cold currents flowing toward the Equator in the deep ocean are often agitated by powerful storms. These disturbances transport huge volumes of sediment across the ocean bottom. Sci. Am. March, 42-53.

Lehfeldt, R., Bloss, S., 1988. Algebraic turbulent model for tidal flows. In: Dronkers, J. (Ed.). Physical Processes in Estuaries, Springer, Berlin, pp. 278-291.

Manning, A.J., Dyer, K.R., 1999. A laboratory examination of floc characteristics with regard to turbulent shearing. Mar. Geol. 160, 147-170.

McCave, I.N., 1976. The Benthic Boundary Layer, Plenum, New York.

Mehta, A.J., 1989. On estuarine cohesive sediment suspension behavior. J. Geophys. Res. 94 (C10), 14 303-14 314.

Perigaud, C., Brun-Cottan, J.C., Biscaye, P., Carson, S., 1982. Resuspension process of sediment from the bottom in the New-York Bight. Euromec 156, The mechanics of sediment transport, 12-14 July 1982.

Sauvaget, P., 1987. Modélisation numérique des écoulements en estuaires et réservoirs. Thèse de Doctorat INPG, 173pp.

Soulsby, R.L., 1983. The bottom boundary layer of shelf seas. In: Johns, B. (Ed.). Physical Oceanography of Coastal and Shelf Seas, Elsevier, Amsterdam (chap. 5).

Stenberg, R.W., Berhane, I., Ogston, A.S., 1999. Measurement of size and settling velocity of suspended aggregates on the northern California continental shelf. Mar. Geol. 154, 43-53.

Syvitsky, J.P.M., Asprey, K.W., Leblanc, K.W.G., 1995. In-situ characteristics of particles settling within a deep-water estuary. Deep-Sea Res. II 42 (1), 223-256.

Taylor, G.I., 1921. Diffusion by continuous movements. Proc. London Math. Soc., Ser. A 20, 196-211.

Taylor, G.I., 1954. The dispersion of matter in turbulent flow through a pipe. Proc. R. Soc. London, Ser. A 223, 446-468.

Thorn, M.F.C., Parsons, J.G., 1980. Erosion of cohesive sediment in estuaries: an engineering guide. Proc. Third Int. Symp on Dredging Tech., pp. 349-358. 